\title{
COMPROMISO DE RETRIBUCIÓN JUSTA EN LOS JURAMENTOS MÉDICOS
}

\author{
Marta L. Pérez * Ana M. Rancich, Ricardo J. GelpI \\ Trabajo realizado en la Facultad de Ciencias Médicas, Universidad Nacional de La Plata y \\ Facultad de Medicina, Universidad Nacional de Buenos Aires, Argentina.
}

RESUMO - Objetivo. Determinar se os Juramentos Médicos de diferentes épocas expressam o compromisso do médico de solicitar uma remuneração justa pelo atendimento e serviços prestados na recuperação da saúde de pacientes.

MétODOs. Foram analisados 50 Juramentos Médicos editados em artigos e publicações, segundo duas épocas diferentes, 12 juramentos da época antiga e medieval e 38 juramentos da época moderna e contemporânea.

Resultados. Somente três textos de juramento, entre os $\mathbf{5 0}$ selecionados, contêm expressamente explícito o compromisso de remuneração justa dos honorários médicos. Dois deles são da época medieval e pertencem à Escola de Medicina de Montpellier, e apenas um da época moderna (Juramento de Amato Lusitano). Quatro fórmulas indicam votos com a atenção dos pobres e II assinalam indiretamente não obter ganhos de outras atividades ou relações.
ConcLusöEs. Os Juramentos mais antigos são os que enfatizam a justa remuneração dos serviços médicos, a atenção médica sem discriminar 0 atendimento em função da possibilidade de pagamento do paciente e a obtenção de ganhos por meios honestos. Os textos de juramentos atuais não expressam estes aspectos, sendo poucos os que se referem somente a não exercer a profissão para proveito material. Apesar destas evidências, os médicos devem respeitar os limites de suas obrigações e estarem comprometidos no sentido de atender pacientes sem discriminar, especialmente sem levar em conta as possibilidades financeiras dos pacientes $e$, ao mesmo tempo, não exigir honorários que não estejam de acordo com os serviços prestados.

Unitermos: Juramentos Médicos. Justiça. Honorários Médicos. Ética Médica.

\section{INTRODUCCIÓN}

Apolo, dios de la salud, se enamora de Coronis, con la que concibe un hijo. A su vez, su amada se casa con Ischys. Al descubrir Apolo la infidelidad hiere mortalmente a Coronis y antes de que muera le arrebata del vientre a su hijo, Asclepio. Se lo entrega al centauro Quirón para que le enseñe el arte de curar. Pronto el discípulo supera al maestro sanando a los enfermos y resucitando a los muertos, con la sangre de la Medusa legendaria que le da Atenea. Dominado por estos poderes y aunque algunos dicen también por amor al oro, se aventura demasiado y abusa de ellos. Hades, el rey de los Infiernos, se queja a Zeus porque Asclepio no mantiene el equilibrio de la población y no respeta los límites de sus obligaciones: no atiende a todos por igual (respeto del derecho a la salud y no discriminación) y además, se excede en su

\section{* Dirección Posta}

Departamento de Autoevaluación Facultad de Ciencias Médicas Universidad Nacional de La Plata Calle 60 y 120, 1900 LA PLATA ARGENTINA

Tel: 54 - 221 - 489-5043 - Fax: 54 - 221 - 4570281 jmarlupe@aol.com codicia (honorarios justos). Por ello, lo castiga aniquilándolo con un rayo'. En definitiva, la causa de la muerte de Asclepio es consecuencia de no haber respetado el principio de justicia.

Este principio como se caracteriza en la actualidad en lo referente al derecho a la salud, a la no discriminación y a la distribución equitativa de recursos, no ha sido una norma dominante en tiempos de Hipócrates y quizás sea la razón de su ausencia en el Juramento Hipocrático ${ }^{2,3}$. Si bien algunos autores consideran que este texto, al expresar "daños voluntarios y actos injustos" hace referencia a este principio ${ }^{4,5}$. De esta forma lo entiende Edelstein que lo adjudica como propio de la Escuela Pitagórica ${ }^{4}$. En cambio, Veatch señala que algunas traducciones del texto griego, sobre todo al inglés, han trasladado el término adiki'é como justicia; siendo en realidad vocablos pertenecientes al principio de no maleficencia: "maldad y daño" y "daños voluntarios y actos perversos"

A pesar de que el Juramento Hipocrático no contempla el principio de justicia, ha sido analizado desde otros puntos de vista, principalmente de los valores morales que sustentan la relación médico-paciente ${ }^{6,7}$, así como también aquellos que se refieren a los aspectos pedagógicos ${ }^{8,9}$. De la misma manera, resulta interesante destacar que en Estados Unidos, Canadá, Reino Unido y Argentina se han realizado estudios con el objeto de determinar el uso de los diferentes Juramentos en las escuelas médicas. En estos trabajos también se analizan las distintas normas señaladas en las diferentes fórmulas. Dos aspectos referidos a esta temática se hallan mencionados: la no

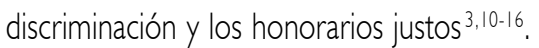

En la actualidad la mala distribución de recursos económicos, el notable aumento de la expectativa de vida y los servicios médicos basados en la alta tecnología agudizan la reflexión sobre la aplicación del principio de justicia en salud. Teniendo en cuenta este principio, el profesional debe comprometerse con el reconocimiento del derecho a la salud que tienen todos los individuos por igual, sin importarle su condición o sus posibilidades de pago (no discriminación), y por ello brindarle toda su habilidad para prevenir la enfermedad y cuando sea necesario ayudarlo a recobrar la salud (principio de beneficencia). Y a su vez, también los médicos son merecedores de un pago por los servicios prestados a los pacientes. Por ello, deben comprometerse a solicitar 
una retribución justa en total concordancia con las características de las actividades realizadas para el logro de la recuperación de la salud ${ }^{17}$.

A pesar de los estudios señalados anteriormente, no se han hallado trabajos que analicen estos aspectos de justicia en los Juramentos Médicos de las escuelas médicas europeas, americanas y orientales y de sociedades profesionales de diferentes épocas ${ }^{2}$, salvo los que hemos efectuado sobre los aspectos pedagógi$\cos ^{8}$, los principios de beneficencia y nomaleficencia $^{18}$ y la regla de confidencialidad ${ }^{19}$. Por ello, resultó de interés analizar la manifestación del compromiso de retribución justa en la relación médico-paciente en Juramentos Médicos de diferente época.

Teniendo en cuenta estos aspectos en el vínculo médico-paciente y dado que el derecho del ser humano a la salud lo analizamos en otro artículo en relación con el principio de beneficencia ${ }^{18}$, el objetivo del presente es determinar si los Juramentos Médicos de diferente época expresan el compromiso de solicitar una retribución justa por las tareas realizadas en la atención médica.

\section{Métodos}

Para el análisis del compromiso de solicitar una retribución justa se estudiaron cincuenta Juramentos Médicos hallados en distintos artículos y publicaciones. Estos textos fueron seleccionados sólo teniendo en cuenta el contexto histórico en que fueron redactados. No se consideraron ni su práctica ni las consecuencias.

En cuanto a la época de formulación, los textos hallados se agruparon en doce antiguos y medievales y treinta y ocho modernos y contemporáneos.

En la tabla I se hallan los nombres y los períodos a los que pertenecen, como asimismo la referencia bibliográfica de donde fue extraído el texto.

Para su análisis, los Juramentos Médicos estudiados se dividieron en los que hacen referencia al compromiso de solicitar una retribución justa por servicios prestados al paciente y en aquéllos, que no lo expresan, teniendo en cuenta las siguientes caracte rísticas:

Compromiso de solicitar retribución u honorarios justos: se refiere al pago acorde con las tareas realizadas por el médico en la recuperación de la salud 45 .

\section{Resultados}

De los cincuenta Juramentos Médicos analizados, sólo tres (6\%) expresan específicamente el compromiso de solicitud de honorarios justos. Los restantes cuarenta y siete (94\%) no lo manifiestan (Tabla I).

Dos de estos tres Juramentos que indican esta obligación son medievales y pertenecen a la Escuela de Medicina de Montpellier, uno usado al finalizar la Licenciatura y otro, al concluir el Doctorado. Es digno de destacar que esta última fórmula manifiesta en forma precisa el compromiso de honorarios justos. Así expresa ${ }^{20,21}$ :

"... y no exigiré jamás un salario que no esté en relación con mi trabajo..."

En cambio, el texto del Juramento de la Licenciatura indica que no exigirá recompensa excesiva. Cabe destacar que ambas señalan que atenderán a los pobres en forma gratuita ${ }^{20-2 !}$.

El otro texto que señala este compromiso es el de Amato Lusitano. Esta fórmula más que un Juramento ha sido caracterizado por ser un testimonio de vida ${ }^{21}$. Lusitano fue un médico hebreo-portugués que escribió manuales de medicina, ejerció en Salónica y, acumuló gran experiencia que la sintetizó en este Juramento. Si bien no habla de retribución justa, señala su moderación en solicitar honorarios ${ }^{21}$ :

"... habiendo tratado a mucha gente con mediana recompensa y mucha otra gratuitamente, muchas veces rechacé firmemente grandes salarios..."

Al igual que los anteriores también destaca la atención gratuita. De la misma forma, es importante señalar que concluye estos conceptos indicando que nunca pretendió21:

"...volverse rico por su liberalidad o por sus dineros..."

Si bien los restantes cuarenta y siete Juramentos Médicos no manifiestan el compromiso de solicitud de retribución justa, cuatro (8\%) del total indican algo similar a lo expresado por los Juramentos de Montpellier en cuanto a la atención de los pobres. Estas fórmulas son de Asaf ${ }^{21}$, de las Escuelas Médicas medievales de Salerno ${ }^{21}$ y de París $^{20}$ y el Compromiso que promueve el bien del paciente ${ }^{36}$.

En cuanto al texto de París se caracteriza por manifestar compromisos relacionados con la actividad del alumno en la Escuela. Por consiguiente, el estudiante se compromete $\mathrm{a}^{20}$ : "...concurrir a las consultas que tienen lugar el mismo día a favor de los enfermos pobres...".

El Compromiso que promueve el bien del paciente expresa que no ejercerá discriminación en la atención aunque los pacientes sean pobres o no puedan pagar. Lo expresa de la siguiente forma ${ }^{36}$ :

"...cuidar a todos los que necesiten mi ayuda con igual interés y dedicación, indepen dientemente de la posibilidad de pagar..."

Aunque son pocos los textos que hablan sobre este compromiso de retribución justa en forma explícita, hay otros que manifiestan, promesas de no buscar otras formas de pago que no sea por su trabajo específico. Así, tres Juramentos (6\%) de los cincuenta hablan de las ganancias, contribuciones o relaciones deshonestas con boticarios (Juramentos del Estatuto de la Medicina y de la Especialización en Venecia ${ }^{24}$, de Salerno ${ }^{21}$ y Doctoral de Basilea ${ }^{26}$.

Otros dos textos (4\%) expresan también no obtener ganancias dando o vendiendo tóxicos o deshonrando sangre inocente para provecho material (Juramentos de Asaf ${ }^{2}$ y del Estatuto de la Medicina y de la Especialización de Venecia $\left.{ }^{4}\right)$.

El Juramento para Doctores en Medicina de la Universidad de Berna ${ }^{28}$ contempla que al ejercer el arte no considerará recompensa alguna. Esta expresión es muy amplia dado que no hace referencia específicamente a una retribución por parte del paciente.

Asimismo, la Paráfrasis Hebrea del Juramento Hipocrático ${ }^{25}$ señala que:

"...devolverá la salud al enfermo, no con esperanza de pago..."

Algo similar expresa con otras palabras el Solemne Juramento de la Universidad de Moscúul', pero pidiendo como recompensa:

"...el amory respeto del pueblo entero..."

Por último, dos Juramentos, uno de la Universidad de Berlín ${ }^{27}$ y otro de la Asociación Médica Británica ${ }^{39}$ destacan que:

"...no ejercerán la medicina solamente para su propio provecho..."

En definitiva, si bien son sólo tres (6\%) de los cincuenta Juramentos Médicos que expresan explícitamente el compromiso de retribución justa, otros once (22\%) señalan indirectamente de una u otra manera, no obtener ganancias o beneficio material de otras actividades o relaciones que no hacen a un justo honorario por servicios prestados para la recuperación de la salud de los pacientes. 
Tabla I - Manifestación del compromiso de retribución justa en los juramentos médicos

\section{RETRIBUCIÓN JUSTA \\ Manifiestan el \\ compromiso}

$n=3(6 \%)$

\section{No maniflestan el compromiso}

$n=47(94 \%)$

\section{JURAMENTOS MÉDICOS}

- de la Escuela de Medicina de Montpellier (Siglo XII o XII ) (Me) ${ }^{20}$

- de la Escuela de Medicina de Montpellier (Siglo XII o XII ) (Me)2I

- de Amato Lusitano (1559) (Mo) ${ }^{21}$

- Hipocrático (Siglo IV a.C) (A) $)^{22}$

- de Caraka (Siglo I d.C.?) (A)22

- de Asaaf (Siglo III a l VII) (Me)2

- de acuerdo con Hipócrates como sería jurado por un cristiano (Siglo X X XI) (Me)

- de la Escuela de Medicina de Salerno (Doctorado) (Siglo XII o XIII) (Me)

- de la Escuela de Medicina de Salerno (Siglo XII o XII) (Me)2I

- de la Escuela de Medicina de París (Siglo XII O XII) (Me)

- del Estatuto de la Medicina en Venecia (I258) (Me)24

- Juramento Arabe (siglo XIII) (Me) ${ }^{23}$

- Paráfrasis Hebrea del Hipocrático (Siglo XV) (Me) ${ }^{25}$

- Doctoral de Basilea (I570) (Mo)26

- de la Universidad de Lovaina (Siglo XVI) (Mo)2

- de la Universidad de Berlín (I810) (Mop)

- para Doctores en Medicina de la Universidad de Berna (1836) (C28

- de los Médicos de Glasgow ( 1868$)(C)^{23}$

- Sponsio Académica de la Universidad de Edimburgo (1873-74, 1919-20) (C99

- para Graduados Médicos de la Universidad de Aberdeen ( 1888 ) (C)

- de los candidatos al grado de Doctor en Medicina (1928) (C)

- de la Universidad de Dalhousie ( 1928) (C)

- de la Universidad de Montreal (1928-1959) (C) ${ }^{10}$

- declaración de Ginebra (1948, con modificaciones 1968 y 1983) (C)22

- de la Universidad Hebrea (1952) (C)²7

- de la Universidad de Ohio (1957-59) (C)

- de la Universidad de Moscú (1963) (C)

- de Louis Lasagna (1964) (C) ${ }^{15}$

- de los Médicos Soviéticos (1971, modificado en 1983) (C)²

- del Colegio de Medicina y Odontología de Nueva Jersey (1976) (C) ${ }^{4}$

- de la Graduación en Medicina de la Universidad Martín Lutero, Halle-Wittenberg (1976) (C³3

- del Médico Musulmán (1977) (C)22

- del Doctor del Código Islámico de Etica Médica. Documento de Kuwait( 1981$)(C)^{22}$

- de la Escuela Johns Hopkins ( 1985$)(C)^{34}$

- del Cantón de Berna (1986) (C)

- Compromiso del médico que promueve el bien del paciente (1988) (C)

- Promesa de los Médicos Rusos (1992) (C)

- de la Universidad de Yale (1993) (C)

- Afirmación de Stanford (1993) (C) ${ }^{15}$

- de Hipócrates modificado. Universidad de California (1993) (C) ${ }^{15}$

- de la Universidad de McGill (1993) (C)15

- de la Universidad George Washington (1993) (C)

- de la Universidad de Ottawa (1993) (C)15

- Redeclaración del Hipocrático (1995) (C) ${ }^{38}$

- Bosquejo de la revisión del Hipocrático (1997) (C) 39

- de la Universidad de Ankara, Turquía (1998) (C) ${ }^{40}$

- Plegaria de la Universidad de Gales (1998)(C)

- dela Universidad de Harvard (1998) (C) ${ }^{42}$

- del Consejo Médico de Singapur (1999) (C) ${ }^{43}$

- de la Universidad de Praga (1999) (C) $)^{44}$ 


\section{Discusión}

En este estudio hemos analizado en los Juramentos Médicos la manifestación del compromiso que hace el propio profesional de requerir una compensación justa, acorde con los beneficios prestados sin exigir más de lo necesario y sin lograr un provecho material más allá de las limitaciones lógicas de su real tarea.

El Juramento Hipocrático, primer documento ético, no hace referencia al principio de justicia en ninguno de sus aspectos, pero Edelstein considera el término "actos injustos" como referido a esta norma ${ }^{4}$, aunque por el contrario, Veatch señala, en realidad, que el original griego expresa "actos dañinos o perjudiciales" (problemas de traducción). A pesar de ello, Edelstein arroga esta característica del Juramento, junto con otras, como perteneciente a la Escuela Pitagórica4 A pesar que no está especificado en el Juramento, Hipócrates señala no ejercer discriminación por la posibilidad de pago del paciente en el libro de los Preceptos. Al respecto expresa ${ }^{23}$ :

"... ser amable, pero considerar cuidadosamente los medios del paciente... y algunas veces dar sus servicios por nada, apelando a una previa donación o a una satisfacción presente. Y si hay oportunidad de servir a alguien extranjero, estrecho de finanzas, dar plenamente asistencia a tal. Donde hay amor al hombre, hay amor al arte ..."

Los tres Juramentos que mencionan el compromiso de solicitud de retribución justa y también de atención gratuita a los pobres son dos medievales y uno, moderno. De los otros tres textos que señalan la asistencia a los indigentes, también dos pertenecen a escuelas médicas medievales y sólo uno es una fórmula contemporánea redactada por una personalidad estudiosa de estos temas éticos. El predominio de este aspecto en los Juramentos medievales puede ser debido a una mayor influencia religiosa, sobre todo cristiana en las escuelas médicas donde han hecho primar el concepto de filantropía ${ }^{46}$. Además, los tres textos medievales hebreos (de Asaf, de Amato Lusitano y Paráfrasis Hebrea del Hipocrático), destacan la atención al pobre y no ejercer con esperanza de ganancias. Esto puede haber sido como lo señalan Kotter, Leibowitz y Richler, una respuesta a las críticas contra los médicos judíos de esos tiempos, por su avaricia. Además, este profesional al igual que el rabino, en la ley hebrea no debían aceptar una recompensa por sus servicios, sólo una compensación por el tiempo perdido, aunque comúnmente se aceptaba el pago de los pacientes ${ }^{24}$.

En cuanto a la prohibición de obtener ganancias por otros medios, también son los textos medievales y modernos los que la expresan. En cambio, sólo dos fórmulas contemporáneas se refieren a no practicar la medicina para su beneficio material. En síntesis, son los Juramentos más antiguos los que enfatizan este compromiso de retribución justa, a la atención sin discriminar por la posibilidad de pago y a la obtención de ganancias por medios honestos, mientras que los textos actuales prácticamente no expresan estos aspectos, siendo muy pocos los que se refieren solamente a no ejercer la profesión para provecho material.

Se han realizado trabajos que estudian el uso de los Juramentos en las escuelas médicas de los Estados Unidos, Canadá y Argenti$n a^{3,10-16}$ y han efectuado, a su vez, un análisis del contenido de los mismos. Sólo cinco de estos hacen referencia a ítems que se relacionan al principio de justicia. Irish y McMurray en un estudio de los Juramentos de las escuelas médicas de Estados Unidos y Canadá indican únicamente la no discri minación por "condición racial, social, etc.", en el 23\% de las fórmulas analizadas". Al hacer un estudio similar Friendlander se refiere a "no interesarse por los honorarios u honores", con un $19 \%$ de manifestación ${ }^{14}$. En una investigación realizada por Dickstein, Erlen y Erlen sobre los principios de beneficencia, no-maleficencia, justicia y respeto por la autonomía del paciente, y las reglas de veracidad y confidencialidad, encuentran casi un 50\% de los textos de escuelas médicas de Estados Unidos que señalan el principio de justicia, destacando algunas promesas con respecto a la no discriminación y a la atención al pobre 3 . Otro trabajo similar es realizado por Orr, Pang, Pellegrino y Siegler con los Juramentos de las escuelas médicas de Estados Unidos y Canadá. Estos autores analizan las fórmulas teniendo en cuenta un estudio del Juramento Hipocrático realizado por Kass ${ }^{47}$. Un aspecto analizado es el referido a justicia, pero expresado en la siguiente forma: "estar libre de toda injusticia voluntaria". El $71 \%$ de los textos de las escuelas médicas lo manifiestan ${ }^{15}$. Cabe destacar una vez más, que esta expresión se puede considerar como "actos perjudiciales", como lo señala Veatch ${ }^{2}$. En un estudio similar al de Dickstein, Erlen y Erlen que realizamos con los Juramentos Médicos de las facultades de medicina de la Argentina, encontramos que el $40 \%$ de los textos analizados mencionan el principio de justicia referido al aspecto de la no discriminación ${ }^{16}$.

Comparando los resultados de estos trabajos con los del presente, concluimos que el porcentaje de manifestación es muy inferior, pero, en primer lugar, debemos reconocer que sólo analizamos el compromiso de retribución justa y en segundo lugar, esta investigación estudia los Juramentos pertenecientes a distintas épocas, a diferencia de los otros que analizan textos de un mismo momento y lugar $^{14}$.

\section{Conclusiones}

En definitiva, podemos concluir que el compromiso de retribución justa, prácticamente no es tenido en cuenta en los Juramentos Médicos actuales, demostrado por este trabajo y los otros analizados. Al respecto, cabe preguntarse por qué este compromiso ha desaparecido de los Juramentos, por qué no se señala más como promesa la atención gratuita a los que carecen de recursos y por qué son muy pocos los textos contemporáneos que expresan no ejercer la Medicina para el beneficio personal. Quizás como señala Smith parece que los Juramentos han tenido una declinación general como respuesta a los variados cambios que ha sufrido la profesión ${ }^{48}$.

Asimismo, es válido también considerar que este aspecto de retribución justa al médico, no sólo debe ser una exigencia por parte del profesional, sino que la sociedad a través de las distintas institu ciones prestadoras deben realmente pagar acorde con los servicios prestados. Esto fue manifestado oportunamente en 1919, por John Round del Battersa Hospital de Londres, al sugerir que el Juramento Hipocrático debía ser actualizado en varios aspectos. Dado que si bien su profesión constituía una gran vocación, no siempre era respetada en términos mo netarios. 
Ejemplificaba este hecho señalando que por las regulaciones de la Policía de Londres pagaban el triple a un veterinario cirujano por curar a un animal herido que un médico por atender a una persona. Indica que la razón de esto es que ${ }^{49}$ :

"...el veterinario cirujano es considerado como un hombre que se gana su vida mientras el médico se supone que existe para el bien público"

En definitiva, a pesar de todo esto, los médicos deben respetar los límites de sus obligaciones y comprometerse a atender sin discriminar, especialmente sin tener en cuenta las posibilidades de retribución de los pacientes y no exigir honorarios que no estén acordes con los servicios prestados.

En conclusión, si los médicos piden como Asclepio a Atenea la sangre de la Medusa para curar aquellos pacientes que pueden retribuir mejor las atenciones recibidas, obligarán a los dioses, por el castigo pedido en la imprecación de los Juramentos Médicos, a que envíen rayos a través de los cielos...

\section{Agradecimiento}

Los autores agradecen a Néstor Alberto Rancich (h) por la traducción del resumen en portugués.

\section{Conflicto de interés: no hay.}

\section{SUMMARY}

\section{Statement of fair retribution in MEDICAL OATHS}

BACKGROUND. to determine if Medical Oaths from different times include the statement of the physician to request from patients a fair retribution for his/her medical services.

Methods. Fifty Medical Oaths found in articles and publications were analyzed. In accordance with their corresponding dates, the Oaths were grouped as ancient/medieval ( 12$)$, and modern/contemporary (38).

RESULTS. Of the fifty, only three specifically included the statement of fair retribution. Two of the three were medieval and belonged to the School of Medicine of Montpellier. The other text was modern (Amato Lusitano's Oath). Four writings showed statements regarding medical assistance to the poor. Eleven pledges indirectly stated that no earnings from other activities and/or relations were obtained.
Conclusions. Ancient oaths emphasize fair retribution, no discrimination in medical assistance based on payment possibilities, and gain of honest earnings. Modern oaths generally do not include these topics and very few mention that the medical profession should not be exercised merely for material purposes. Despite the above, physicians should respect the limits of their obligations and should be committed to assist without discriminating, particularly without taking into consideration their patient's financial possibilities. Therefore their fees should not be excessive for the services rendered. [Rev Assoc Med Bras 2004; 50(3): 338-43]

KeY words: Medical oaths. Justice. Medical fees. Medical ethics.

\section{ReferenCiAs}

I. Haggard H. El Médico en la historia. $3^{\mathrm{a}}$ ed. Buenos Aires: Editorial Sudamericana; 1946. p.67-8.

2. Veatch RM. Medical codes and oaths. In: Reich TW, editor. Encyclopedia of bioethics. $2^{\text {nd }}$ ed. New York: The Free Press. A Division of McMillan Publishing Co. Inc; 1995. p. I4 I9-35.

3. Dickstein E, Erlen J, Erlen JA. Ethical principles contained in currently professed medical oaths. Acad Med 1991; 66:622-4.

4. Edelstein L. The Hippocratic oath: text, translation and interpretation. In: Veatch R, editor. Cross cultural perspectives in medical ethics: readings. Boston: Jones and Bartlett Publishers; 1989. p.6-24.

5. Thompson IE. Fundamental ethical principles in health care. BMJ 1987; 295: | 46 |-5.

6. Bonds C. The Hippocratic oath: a basis for modern ethical standards. JAMA 1990; 264:231I.

7. Goic CA. El juramento Hipocrático: ¿una veneración ciega? Bol Of Sanit Panam 1993; | | 5: | 40-6.

8. Gelpi R; Rancich A. Aspectos educativos en los Juramentos Médicos. Medicina (B Aires) I 984; 44:430-2.

9. Reiser $\mathrm{S}$. The ethics of learning and teaching. Acad Med 1994; 69:872-6.

10. Carey E. The formal use of the Hippocratic Oath for medical students al commencement exercises. Bull Am Assoc Med Coll 1928; 3:159-66.

I I. Irish D; McMurray D. Professional Oaths and American Medical College. J Chronic Dis 1965; 18:275-89.

12. Crawshaw R. The contemporary use of medical oaths. J Chronic Dis 1970;23: I 44-50.

13. Crawshaw R; Link C. Medical Oaths: a contemporary perspective. West J Med 1996; 1 64:452-6.

14. Friedlander WJ. Oaths give by US and Canadian medical school 1977 profession of values. Soc Sci Med 1982; 16:1 15-20.
15. Orr RD, Pang N, Pellegrino ED, Siegler M. Use of the Hippocratic Oath: a review of twentieh century practice and a content analysis of oaths administered in medical schools in the U.S. and Canada en 1993. J Clin Ethics 1997; 8:377-88.

16. Rancich A, Gelpi R. Juramentos médicos utilizados en las facultades de medicina de Argentina en relación al Hipocrático. Medicina (B Aires) 1998; 58: 1 47-52.

17. Roncoroni AJ. La Ética Médica en el mundo del mercado. Fidelidad Hipocrática o fidelidad a la empresa. Medicina (B Aires) 2000; 60:83-88

18. Rancich AM, Pérez ML, Gelpi RJ, Mainetti JA. Principios de beneficencia y no-maleficencia en los Juramentos Médicos de diferentes épocas y orígenes. Rev Argent Cardiol 2000; 68:30 I-8.

19. Gelpi RJ, Pérez ML, Rancich AM, Mainetti JA. Confidencialidad en los Juramentos Médicos (Cuando el cuervo blanco se vuelve gris...) Medicina (B Aires) 2000; 60:506-4.

20. Díaz Trigo A. El Juramento en las Universidades de París y Montpellier. Rev Assoc Méd Arg 1958; 72:498-50।.

21. De Pina L. Juramentos médicos. A propósito de oração ética de Assafe. Lisboa: Ed. Da Imprensa Médica; 1952. p.13-5, 17-8,235,27-30,38.

22. Codes, oaths and directives related to bioethics. In: Reich WT, editor. Encyclopedia of bioethics. $2^{\text {nd }}$ ed. New York: The Free Press. A Division of McMillan Publishing Co. Inc; 1995. Appendix, Section II, p.2630-706.

23. Jones W. The Doctor's Oath: an essay in the history of medicine. London: Cambridge University Press; 1924. p.23, 25.

24. Agrimi J., Crisciani C. Malato, medico e medicina nel Medioevo. Turin; 1980. p.208-11.

25. Kotter S; Leibowitz J; Richler B. Hebrew paraphrase of the Hippocratic Oath (from a fifteenth-century manuscript). Med Hist 1978; 22:438-45

26. Medizinhistorisches Institut der Universit Bern Burckhardt, 1970, S. 368f./ S.383f.

27. Bird LP, Barlow J, editors. Codes of medical ethics, oaths, and prayers: an anthology. Richardson(TX): Christian Medical and Dental Society; 1989. p.44, 50.

28. Facultad de la Universidad de Berna. Protocolo. I, S 24 F, 1836.

29. Edinburg University Calendar 1919-1920. Edinburg: Published for ghe University by James Thin, publisher and bookseller. 1919.

30. Pennington T, Pennington $C$. The Hippocratic Oath is not administred in the strictly legal sense [letter]. BMJ 1994; 309:952.

31. Etziony MB. An anthology of medical prayers, oaths and codes of ethics written and recited by medical practitioners through the ages. Sprinfield (ILL): Charles Thomas; 1973. p.96.

32. Cassel C, Jameton A, Sidel W, Storey P. The Physician's Oath and the prevention of nuclear war. JAMA 1985; 254:652-4.

33. Solemn V. Of those graduating in the field of Medicine at the Martin-Luther University, Halle-wittenberg (German Democratic Republic, 1976). J Med Philos 1989; | 4:351. 
34. McHugh P. Hippocrates à la mode. Nature Med 1996: 2:507-9.

35. Government Concuil of Berna Canton. Oath of medical doctor of Berna University. Decree. Switzerland; 1986.

36. Pellegrino E, Thomasma D. A Medical Oath for the Post-Hippocratic Era. In: Pellegrino E; Thomasma D. For the patient's good: the restoration of beneficence in health care. New York: Oxford University Press; 1988. p. 203-6.

37. Tichtchenko P. Resurrection of the Hippocratic Oath in Russia. Camb Q Health Ethics 1994; 3:49-5 |

38. Value of Life Committee, Brigthon, U.S.A. A.D. 1995 Restatement of the Oath of Hippocrates (Circa 400 B.C.). Cuad Bioética 1996; 25: 123-4.

39. Hurwitz B, Richardson R. Swearing to care: the resurgence in medical oaths. BMJ 1997; 3|5:167|-4.

40. Medical Faculty. University of Hacettepe, Ankara, Turkey. Medical Oath, 1999 [online]. Available from: http://www.ankara.edu.tr/ faculties.
4I. Medical College. University of Gales. Pledge to graduates. 1998 [online]. Available from: http:/ /www.uwcm.ac.uk.

42. Harvard Medical School. Oath of Class 1998 [online]. Available from: http://www.hms. harvard.edu Consejo Médico de Singapur. Juramento del Médico. 1999. www.sma.org.sg.

43. Universtiy of Charles. Praga, Czechoslovakia. Oath of Medical Doctor. 1999 [online]. Available from: http://www.cuni.cz/welcome.edu.

44. Beauchamp TL, Childress JF. The principle of nonmaleficence and the principle of beneficence. In: Principles of biomedical ethics. $3^{\text {er }}$ ed. New York: Oxford University Press; 1989. Cap.4, p.106-47, Cap.5, p. $148-82$.

45. Galvao Sobrinho CR. Hippocratic ideals, medical ethics, and the practice of medicine in the early middle ages; the legacy of the Hippocratic Oath. J Hist Med Allied Sci 1996; 51:438-55.

46. Kass LR. Is there a medical ethic: the Hippocratic Oath and the sources of ethical medicine. In Toward a more Natural
Science. New York, Free Press, 1985. p224-46. Citado por Orr RD, Pang N, Pellegrino ED, Siegler M. Use of the Hippocratic Oath: a review of twentieh century practice and a content analysis of oaths administered in medical schools in the U.S. and Canada en 1993. J Clin Ethics 1997;8:377-88

47. Smith DC. The Hippocratic Oath and modern medicine J Hist Med Allied Sci 1996; 51:484500.

48. Round J. The dawn of a medical union. Western Medical Times, 1919:39:429-36. Citado por Smith DC. The Hippocratic Oath and modern medicine. J Hist Med and Allied Sci |996;51:484-500

Artigo recebido: 05/02/2003 Aceito para publicação: 28/I I/2003 